\title{
Erratum: Exploring spiral defect chaos in generalized Swift-Hohenberg models with mean flow [Phys. Rev. E 84, 046215 (2011)]
}

\author{
A. Karimi, Zhi-Feng Huang, and M. R. Paul
}

(Received 7 February 2019; published 7 March 2019)

DOI: 10.1103/PhysRevE.99.039901

In this paper, our numerical results were not plotted correctly in Fig. 7(a). The corrected Fig. 7(a) shows that the spatial variation of the mean flow is a vortex structure near the spiral core and is not a quadrupole as we described in the original paper. Our numerical results yield this vortex structure of the mean flow around spiral cores for both the generalized Swift-Hohenberg equation [1] and Manneville's model [2]. These model equations correctly capture the basic structure of the mean flow near spiral cores. This is evident when comparing Fig. 7(a) with Fig. 7(b) of the original paper which shows the spatial variation of the mean flow around a spiral for the Boussinesq equations. Our plotting error in Fig. 7(a) does not change any of the other results, figures, or conclusions in the original paper.

We would like to thank J. Viñals and E. Vitral for bringing this to our attention.

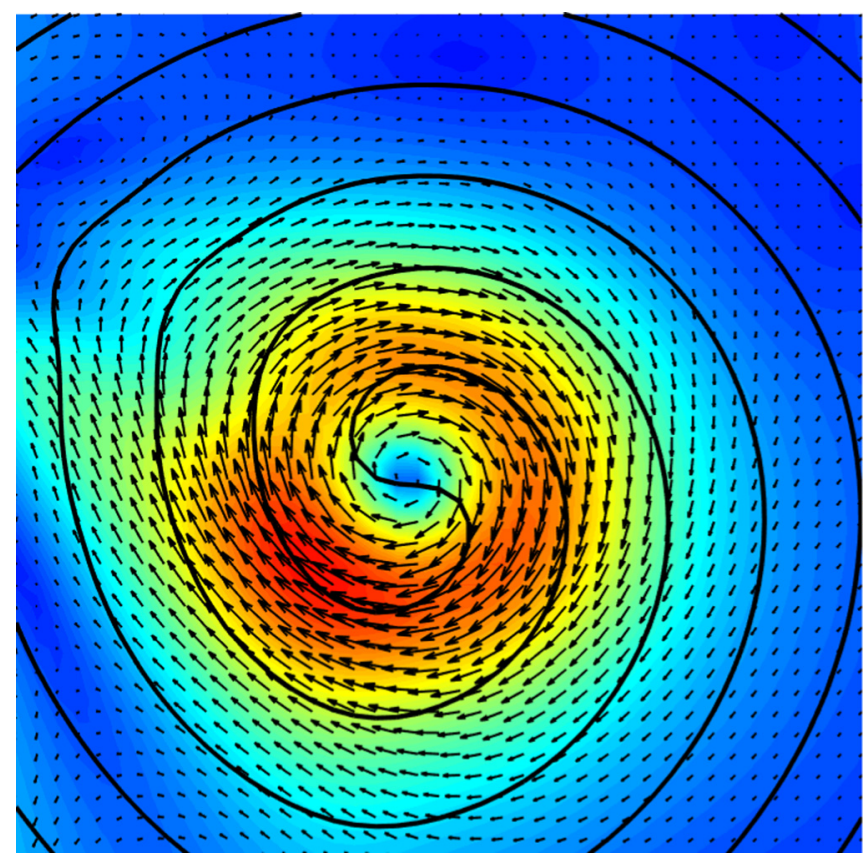

FIG. 7. (a) The spatial variation of the mean flow near a spiral defect in a chaotic pattern. The color contours represent the magnitude of the mean flow, the black lines indicate the convective roll boundaries, and the arrows are vectors for the mean flow. These results are computed using Manneville's equations [2] with system parameters $\epsilon=0.7, \sigma=2, g_{m}=50$, and $c^{2}=0.4$ at time $t=7 \times 10^{5}$.

[1] H. S. Greenside and M. C. Cross, Phys. Rev. A 31, 2492 (1985); H. W. Xi, J. D. Gunton, and J. Viñals, Phys. Rev. Lett. 71, 2030 (1993).

[2] P. Manneville, in Cellular Structures in Instabilities, edited by J. Wesfreid and S. Zaleski (Springer-Verlag, Berlin, 1984), Vol. 210, pp. 137-155; J. Phys. 44, 759 (1983). 